\title{
On estimating the reduction factor of bridge piers
}

\author{
Andrei Benin ${ }^{1}$, Olga Nesterova ${ }^{2}$, Alexander Uzdin ${ }^{3,}$, Sergei Prokopovich ${ }^{4}$, Yuri Rutman ${ }^{5}$, \\ Youhai Guan ${ }^{6}$ \\ ${ }^{1,3,4}$ Emperor Alexander I St. Petersburg State Transport University, Moskovsky ave., 9, Saint- \\ Petersburg, 190031, Russia \\ ${ }^{2,5}$ Saint Petersburg State University of Architecture and Civil Engineering, Vtoraya \\ Krasnoarmeiskaya str, 4, Saint Petersburg, 190005, Russia \\ ${ }^{3,6}$ China University of Petroleum Huadong, Changjiang West Road, 66, Huangdao District, Qingdao, \\ Shandong, 266580No, P.R. China
}

\begin{abstract}
Estimating the reduction factor for calculating massive reinforced concrete bridge piers was made. For this purpose a quasi-static "force-displacement" diagram was built up using the ANSYS software. This diagram has the form of a bilinear one, and the character of the bilinearity depends on the diameter of the reinforcing bars insignificantly. The percentage of reinforcement affects only the moment when all reinforcement bars begin to flow. The reinforcement flow takes place in the displacement interval from 3 to $5 \mathrm{~cm}$. The collapse will occur when the reaction of the bearing part goes beyond the pier cross-section at pier displacements from 5 to $20 \mathrm{~cm}$. Using "force-displacement" diagram, the behavior of the single-mass model with a bilinear deformation diagram and the limit displacement of $20 \mathrm{~cm}$ was analyzed. Then, it became possible to obtain for each accelerogram the limit elastic displacement and the limit position of the point corresponding to the maximum structure displacement during structure oscillations. It was done using real accelerograms of earthquakes with intensity 9 on the MSK scale without normalizing their amplitudes. In this case, long-period accelerograms had smaller peak accelerations, but resulted in greater plastic deformations. As a result, no evident dependence of plastic deformation on the input spectral composition was found and the value of reduction factor K1 turned out to be $0.25-0.27$. However, it is shown that this reduction factor cannot be used to make transition from seismic loads obtained on the basis of timehistory analysis by accelerograms to design loads.
\end{abstract}

\section{Introduction}

Currently, the transition to multi-level designing of seismic structures is taking place in earthquake engineering. In this connection, the notion of the reduction factor is used to relate the results of elastic calculations with those of inelastic ones. This factor is designated as K1 in the Russian Federation Guidelines in use (according to Russian State

\footnotetext{
*Corresponding author: uzdin@mail.ru
} 
Standard "SP 14.13330.2018 Construction in seismic areas. The updated edition of Guidelines II-7-81*"). A great number of works in Russia [1, 2] (Oizerman V.I., Calculation of structures for seismic actions by the method of limiting states, Abstract information / TsINIS. Ser. XIV. Seismic resistant construction, Vol. 9 (1978) 4-7; Rutman Yu. L., Simbort E., The choice of the reduction factor of seismic loads on the basis of analysis of the plastic design resource, Herald of Civil Engineers (2)27 (2011) 78-81) and other countries [3-8] are devoted to the evaluation of the reduction factor. However the main features of estimating this factor are often treated differently, especially in Russian publications. In a lot of studies the coefficient $\mathrm{K}_{1}$ is regarded as the ratio of elastic displacements to the maximum calculated displacements of the elastoplastic system in timehistory calculating using earthquake accelerograms. In this case, the value of K1 depends essentially on the input type. It was established in [5] that for fixed peak ground accelerations (PGA), the values of K1 decrease greatly with the increase of the prevailing input period. However, to use the fixed normative value of PGA as a basis seems completely wrong. The values of PGA depend significantly on the prevailing period of excitation, which is discussed in detail in literature [9-11].

The lack of an unambiguous understanding of the meaning of the reduction factor and methods of it estimating required that the authors should explain their approach to evaluating the reduction factor.

Figure 1 shows a typical diagram of the system monotonic loading. The curve presented on the diagram is called a "push-over curve". 4 points are located on the displacement axis u:

- $u_{e l}$ is the displacement elastic limit;

- $u_{\text {red }}$ is the redundancy point, i.e. the limit of exhaustion of the reserve capacity of the system due to its static indeterminacy, in other words the point of the system transformation into a mechanism;

- $u_{\text {calc }}$ is the calculated displacement of the nonlinear system at a given input;

- $u_{u l t}$ is the maximum system displacement, after which the system will completely collapse. points

On the seismic response axis, the indicated displacements correspond to the following

- $S_{e l}$ is the load elastic limit;

- $S_{\text {lim }}$ is the redundancy point;

- $S_{\text {cond }}$ is the conditional response of the linear system;

- $S_{u l t}$ is the limiting conditional response of the linear system, after which the system collapses.

In addition to the points indicated on the S-axis, the linear system response $S_{c a l c}^{(e l)}$ to the input causing the displacement $u_{\text {calc }}$ in the nonlinear system is shown.

There are two other additional points $S_{G L}$ and $S_{d e s}$ on the S-axis. $S_{G L}$ is the limit load in accordance with the Guidelines in law and $S_{\text {des }}$ is a design load which is compared with the load elastic limit in order to estimate the structure seismic stability.

Note that the $S_{\text {cond }}$ and $S_{\text {ult }}$ values are conditional and are the projection of the corresponding displacements on the elastic response line. 


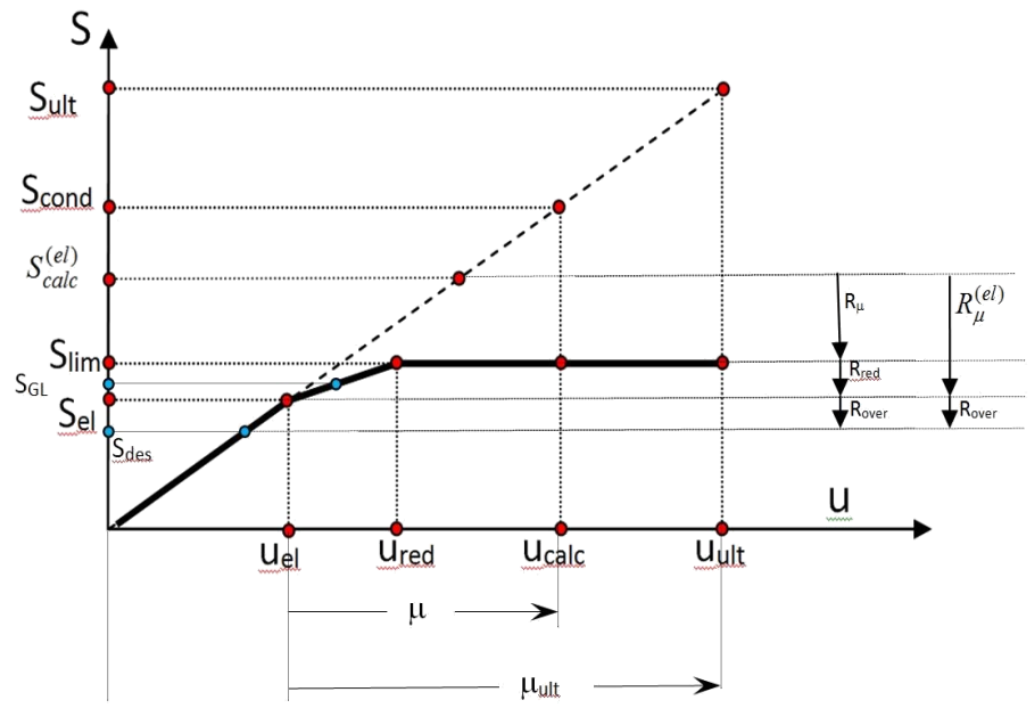

Fig.1. Characteristic points on the diagram of a structure monotonic loading

In the most studies, the following inequality is considered as a condition of seismic stability

$$
S_{\text {des }}<S_{\text {lim }}
$$

In this case, the reduction factor $K_{l}$ reduces the load calculated for the elastic system to the design load $S_{\text {des }}$

$$
S_{\text {calc }}^{(e l)} \cdot K_{1}=S_{d e s}
$$

To estimate the factor $K_{l}$, three auxiliary factors are used

$$
K_{l}=R_{\mu} \cdot R_{\text {red }} \cdot R_{\text {over }}
$$

where $R_{\mu}$ is the response modification factor, which reduces the load $S_{c a l c}^{(e l)}$ to the limit load $S_{\text {lim }}$;

$R_{\text {red }}$ is the factor of static uncertainty that reduces the load $S_{\text {lim }}$ to the elastic limit $S_{e l}$;

$R_{\text {over }}$ is the overload factor that reduces the elastic limit $S_{e l}$ to the design load $S_{\text {des }}$.

These factors are explained in Fig.1

In addition to the three mentioned coefficients $R_{\mu}, R_{\text {red }}$ and $R_{\text {over }}$, coefficient $R_{\mu}$, is used in a number of works, in order to take into account the fact that the calculation is performed with a conditional damping different from the actual one.

Unlike the above mentioned approach, we proceed from the fact that calculating a structure under the MDE is kinematic, since in the ultimate state the strength requirements are violated and the system turns into a mechanism. Then the seismic stability requirement is written in the following form 


$$
u_{\text {calc }}<u_{u l t}
$$

Let us introduce the concept of the limit plasticity factor into the analysis

$$
\mu_{u l t}=\frac{u_{u l t}}{u_{e l}}=\frac{S_{u l t}}{S_{e l}} .
$$

Note that the conventional plasticity factor is defined as

$$
\mu=\frac{u_{\text {calc }}}{u_{e l}}=\frac{S_{\text {cond }}}{S_{e l}}
$$

The value of $\mu$ tends to $\mu_{u l t}$, if the calculated value of the displacement tends to the limit value.

Taking into account (5) inequality (4) can be written in the following form

$$
u_{\text {calc }}<u_{\text {el }} \mu_{u l t}
$$

This inequality can be presented using the above described loads

$$
S_{\text {cond }}<S_{e l} \mu_{u l t}
$$

The Guidelines provide for one the overload of structural elements. If we denote the permissible load by $S_{G L}$, the following expression is obtained

$$
S_{e l}=\kappa \cdot S_{G L},
$$

where the coefficient $\kappa<1$.

As a result, condition (7) takes the following form

$$
S_{\text {cond }}<S_{G L} \cdot \kappa \cdot \mu_{u l t}
$$

An other problem is to present the value of $S_{\text {cond }}$ by the value of $S_{c a l c}^{(e l)}$. To do this, it is necessary to use the reaction modification factor

$$
R_{\mu}^{(e l)}=\frac{S_{e l}}{S_{c a l c}^{(e l)}} .
$$

The factor $R_{\mu}^{(e l)}$ reducing the design response to the elastic response is used in this formula. 
Note that in the accepted notation $R_{\mu}^{(e l)} \neq \frac{1}{\mu}$, as the value of $R_{\mu}$ refers to forces and the value of $\mu$ to displacements.

It should also be noted that the factor $R_{\mu}$ entering formula (3) coincides with the coefficient $R_{\mu}^{(e l)}$ only in the absence of redundancy section, i.e. at $S_{e l}=S_{\text {lim }}$ or, in other words, in the case, when the law of deformation is described by the Prandtl diagram.

The well known energy estimate of the value of $R_{\mu}$ obtained by N.Newmark [12] who used the Prandtl diagram as the deformation one, has the following view:

$$
R_{\mu}=\frac{1}{\sqrt{2 \cdot \mu-1}}
$$

In general, the value of $R_{\mu}$ must depend on the type of input and the diagram of the system loading.

Now, one can write

$$
S_{\text {calc }}^{(e l)}=\frac{S_{\text {cond }}}{\mu \cdot R_{\mu}} .
$$

From here

$$
S_{\text {cond }}=S_{\text {calc }}^{(e l)} \cdot \mu \cdot R_{\mu}
$$

After substituting (14) into (11), we obtain

$$
S_{\text {calc }}^{(e l)} \mu \cdot R_{\mu}<S_{G L} \cdot \kappa \cdot \mu_{u l t}
$$

or

$$
S_{\text {calc }}^{(e l)} \frac{\mu \cdot R_{\mu}}{\kappa \cdot \mu_{u l t}}<S_{G L}
$$

Here the multiplier $\frac{\mu \cdot R_{\mu}}{\kappa \cdot \mu_{u l t}}$ is the reduction factor $K_{l}$.

Using the approach developed in the world literature, one can transform formula (2) with allowance for (3) in the following form 


$$
S_{\text {calc }}^{(e l)} \cdot R_{\mu} \cdot R_{\text {red }} \cdot R_{\text {over }}<S_{e l}
$$

If the normative value $S_{G L}$ is taken in place of (17), then conditions (16) and (17) are satisfied under the condition

$$
\frac{\mu \cdot R_{\mu}^{(e l)}}{\mu_{u l t}}=R_{\mu} \cdot R_{\text {red }} \cdot R_{\text {over }}
$$

if we take into account that $R_{\mu} \cdot R_{\text {red }}=R_{\mu}^{(e l)}$, we get $\frac{\mu}{\mu_{u l t}}=R_{o v e r}$.

Below, the reduction factor is analyzed from the positions of formula (16), i.e.

$$
K_{1}=\frac{\mu \cdot R_{\mu}}{\kappa \cdot \mu_{u l t}}
$$

with respect to the assessment of pier seismic resistance typical of transport construction in Russia.

\section{Materials and methods}

The authors consider a detailed finite element model of the bridge pier body widely used in Russia in transport construction practice. The diagram and the finite-element model of the pier are shown in Figs. 2 and 3, respectively.

Finite element modeling was carried out using the ABAQUS-soft complex [13]. To simulate concrete, three-dimensional linear isoparametric finite elements C3D8R were used; to simulate the reinforcement, beam elements with cubic interpolation B33 were used. The interaction was modeled with the help of the "embedded region" due to the automatic setting of kinematic relationship on the translational degrees of freedom between the nodes of the "host elements" (concrete) and the nodes of the "embedded elements"(reinforcement). The boundary conditions were the prohibition of all displacements in the pier bed. The loads were applied to the pier in two steps: first, the vertical operating (pressure on the upper faces), then, lateral movement at the top point of the pier.

To describe the nonlinearity of the pier behavior, steel reinforcement was simulated using the flow theory with linear isotropic hardening, and concrete was simulated using an elastoplastic model with damage [5] (Rutman Yu. L., Simbort E., The choice of the reduction factor of seismic loads on the basis of analysis of the plastic design resource, Herald of Civil Engineers (2)27 (2011) 78-81). The determining equations, methods for determining constants and calibration of the model are described in detail in $[3,4,6,7]$. Deformation diagrams for reinforcement of class A400 with the design resistance $400 \mathrm{MPa}$ and concrete of classes B30 with the limit prism strength 22MPa (contour blocks) and B10 with the prism strength $7.5 \mathrm{MPa}$ (monolithic core and seams) used in the calculations are shown in Fig. 3.

An attempt at estimating the reduction factor for calculating massive reinforced concrete bridge piers was made earlier by the authors (L.N. Smirnova, A.V. Benin, S.G. 
Semenov, Yu. Guan, Estimation of the reduction coefficient for the calculation of bridge supports, Seismic resistant construction. Safety of structures 6 (2016) 15-19), using the software ANSYS. In this paper a quasi-static "force-displacement" diagram was built up to the point when all the concrete reinforcement "flowed" in the pier body, i.e. the pier became a mechanism. This diagram is presented in Fig.4 for different percentages of the pier reinforcement, and the moment of exhaustion of the bearing capacity of the pier is marked by a red dot. In the paper under consideration the coefficient $\mathrm{K}_{1}$ obtained as the ratio of the elastic displacement to the limit value.

$1-1$

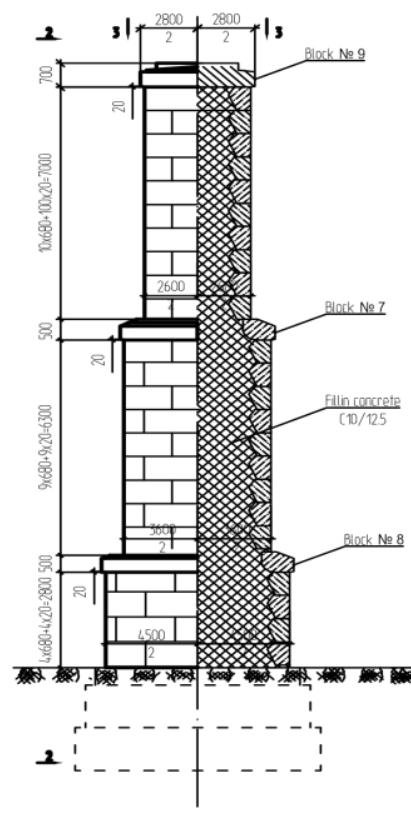

3-3

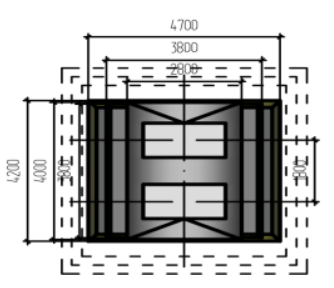

4-4
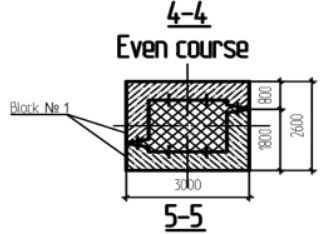

Odd course

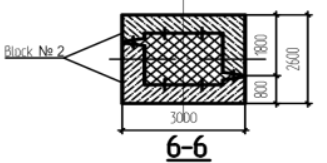

Even course

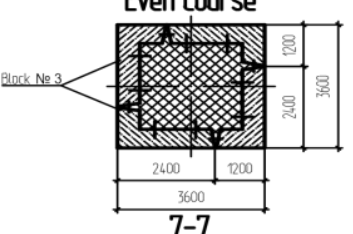

7-7

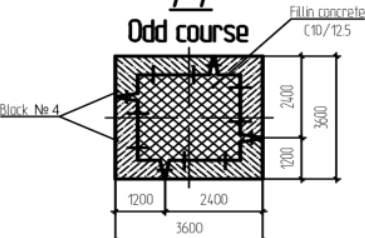

$\underline{8-8}$

Even course

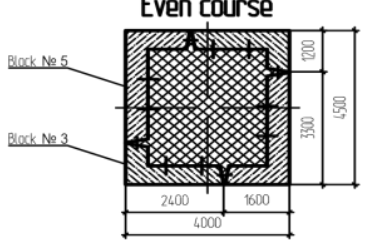

9-9

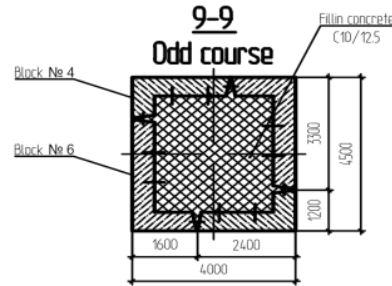

Fig.2. The pier diagram

The dependence obtained allows us to estimate only the Rred factor, which enters into formula (3) to determine the value of $\mathrm{K}_{1}$. The corresponding calculations are given in Table 1. In fact, the pier can keep moving after the moment when the reinforced bars begin to flow without any collapse. The collapse occurs when the reaction of bearings $\mathrm{R}$ extends 
beyond the cross-section of the pier (Fig. 5). This makes it possible to increase the limit movement of the pier up to $20 \div 30 \mathrm{~cm}$.

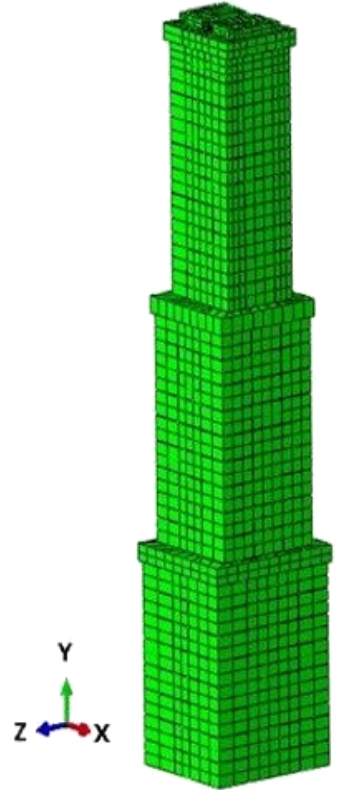

a)

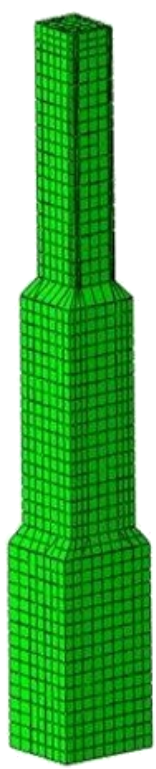

b).

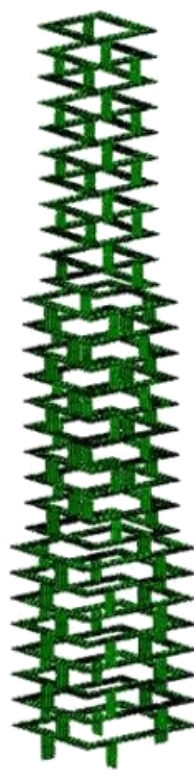

c)

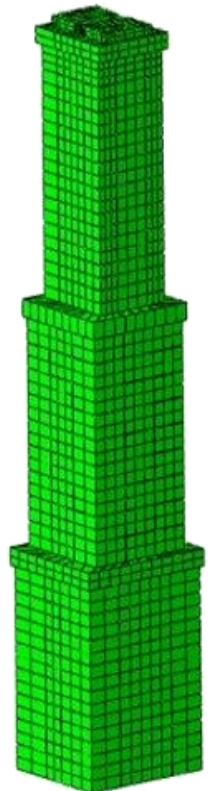

d)

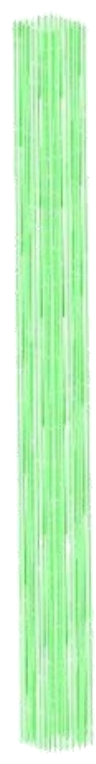

e)

Fig.3. The finite element model of the pier a) the complete model; b) the monolithic core; c) the seams; d) the contour blocks; e) the reinforcement

a)

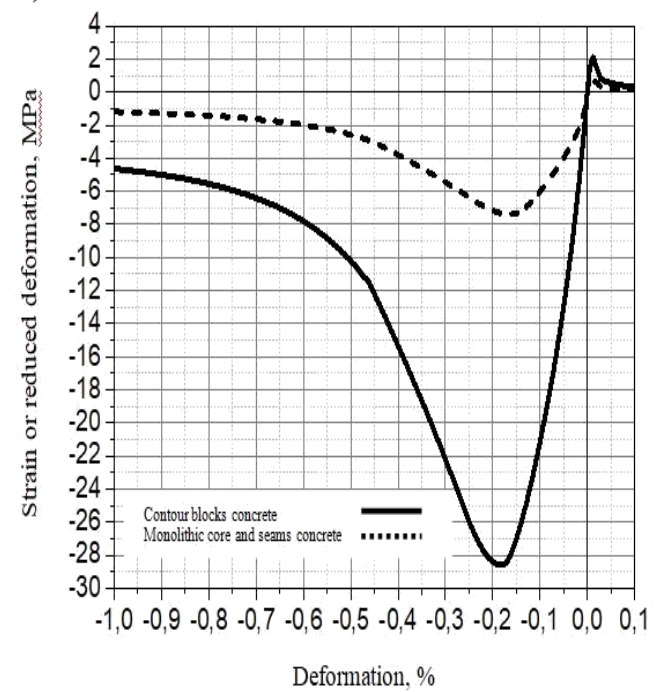

b)

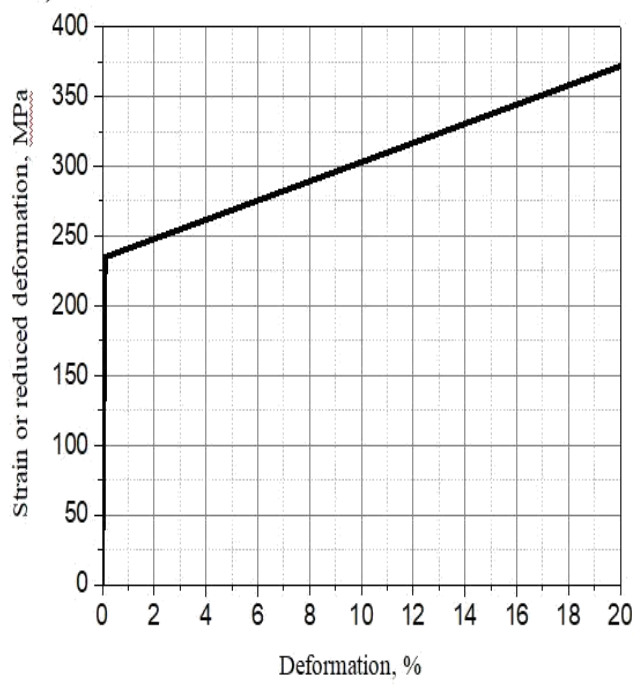

Fig.4. Deformation diagrams of concrete (a) and steel (b) 
If we follow the principles of multilevel designing using maximum design earthquakes, which consider structure calculation as kinematic one, then in the GL formulas for calculating piers it is necessary to use $K_{l}=K_{1}^{(\mathrm{lim})}$. Such decision is justified by the experience of massive bridge pier damages, at which piers remained operational even when the solidity of the pier body was broken (A.A. Nikitin, A.Ju. Simkin, A.M. Uzdin, Engineering Analysis of Spitak Earthquake Effects and Problems for Strengthening Buildings, Proc. of the 9th European Conference on Earthquake Engineering, Vol. 9 (1990) 118-128). For lightweight reinforced concrete piers with a large percentage of reinforcement, such a decision would be erroneous.

Taking into account the above mentioned, we can estimate the value $K_{1}^{(\mathrm{lim})}$ under the assumption that for the most dangerous accelerograms $u_{\text {calc }} \approx \boldsymbol{u}_{\text {ult }}=200 \mathrm{~mm}$. If we assume an overload factor of 1.1, i.e. $\quad R_{\text {over }}=0.909$, then formula (3) gives an estimate of $K_{l}$ shown in the penultimate row of table 1 .

Table 1.The results of the analysis of the monotonically loaded support diagram

\begin{tabular}{|c|c|c|c|}
\hline $\begin{array}{c}\text { reinforcement } \\
\text { percentage }\end{array}$ & $\mathrm{d}=16[\mathrm{~mm}]$ & $\mathrm{d}=24[\mathrm{~mm}]$ & $\mathrm{d}=40[\mathrm{~mm}]$ \\
\hline \multicolumn{4}{|l|}{ characteristics } \\
\hline $\mathrm{u}_{\mathrm{el}},[\mathrm{mm}]$ & 10 & 10 & 10 \\
\hline $\mathrm{S}_{\mathrm{el}},[\mathrm{MN}]$ & 1.75 & 1.75 & 1.75 \\
\hline $\mathrm{u}_{\mathrm{red}},[\mathrm{mm}]$ & 60 & 70 & 88 \\
\hline $\mathrm{S}_{\mathrm{lim},},[\mathrm{MN}]$ & 3.15 & 3.4 & 3.7 \\
\hline $\mathrm{R}_{\mathrm{red}}=\mathrm{S}_{\mathrm{el}} / \mathrm{S}_{\mathrm{lim}}$ & 0.555 & 0.515 & 0.473 \\
\hline $\mathrm{S}_{\mathrm{GL}},[\mathrm{MN}]$ & 2.25 & 2.32 & 2.40 \\
\hline$\kappa=\mathrm{S}_{\mathrm{el}} / \mathrm{S}_{\mathrm{GL}}$ & 0.778 & 0.754 & 0.729 \\
\hline $\mathrm{u}_{\mathrm{ult}},[\mathrm{mm}]$ & 200 & 200 & 200 \\
\hline$\mu_{\mathrm{ult}}=\mathrm{u}_{\mathrm{ult}} / \mathrm{u}_{\mathrm{el}}$ & 13.3 & 13.3 & 13.3 \\
\hline$\mu_{\mathrm{ult}, \mathrm{red}}=\mathrm{u}_{\mathrm{ult}} / \mathrm{u}_{\mathrm{red}}$ & 3.33 & 2.86 & 2.27 \\
\hline $\mathrm{R}_{\mu, \mathrm{ult}}=1 / \sqrt{ } 2 \mu_{\mathrm{ult}}-1$ & 0.198 & 0.198 & 0.198 \\
\hline$R_{\mu, \text { ult,red }}=1 / \sqrt{2} \mu_{\text {ult,red }}-1$ & 0.42 & 0.46 & 0.531 \\
\hline $\mathrm{R}_{\mu, \mathrm{ult}, \mathrm{red}} \cdot \mathrm{R}_{\mathrm{red}}$ & 0.233 & 0.237 & 0.251 \\
\hline $\begin{array}{c}\mathrm{K}_{1}^{(\lim )}= \\
\mathrm{R}_{\mu, \mathrm{ult}} \cdot \mathrm{R}_{\mathrm{red}} \cdot \mathrm{R}_{\mathrm{over}} \\
\text { according to the } \\
\text { formula (3) }\end{array}$ & 0.212 & 0.216 & 0.229 \\
\hline $\begin{array}{c}\mathrm{K}_{1}{ }^{(\lim )}=\mathrm{R}_{\mu, \mathrm{ult}} / \kappa \\
\text { according to the } \\
\text { formula (19) }\end{array}$ & 0.254 & 0.263 & 0.272 \\
\hline
\end{tabular}




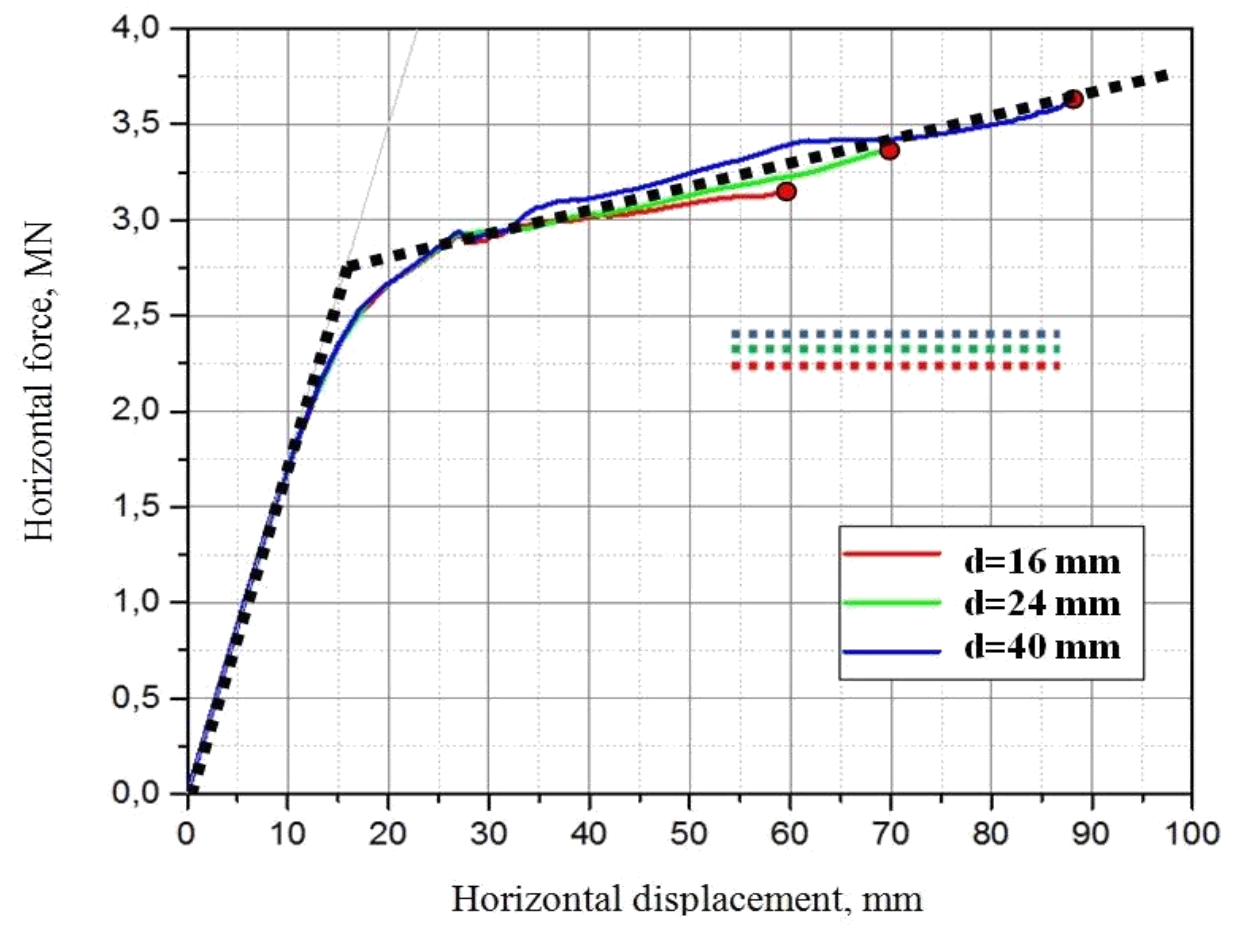

Fig.5. The displacement-force diagrams for the bridge pier and various diameters $d$ of the reinforcement bars. The color dotted lines show the limit forces according to the GL; the dotted bold line shows the load bilinear approximation of the pier displacement-force diagram

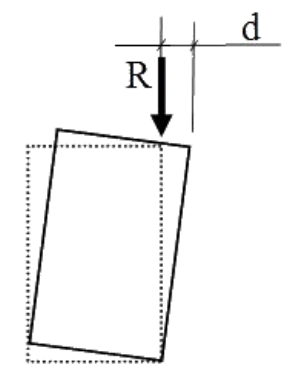

Fig.6. Scheme for estimating the ultimate pier displacement

\section{Estimating the reduction factor on the basis of dynamic time- history calculations}

Keeping the above mentioned in mind, one can use the push-over method [14] to estimate the reduction factor and consider the behavior of a single-mass model with a bilinear deformation diagram and with the limit displacement of $20 \mathrm{~cm}$. The deformation diagram of such model is shown in Fig. 1 by a dotted bold line.

In [5], the author used a package of accelerograms (according to Russian State Standard "SP 14.13330.2018 Construction in seismic areas. The updated edition of Guidelines II-7$81^{*}$ "), assuming the peak accelerations to be fixed. In our opinion, this approach is 
erroneous. If seismic inputs should be normalized, then, in order to characterize the strength of the earthquake, it is necessary to use energy characteristics, which are substantiated in $[10,15,16]$.

The authors of this paper used records of past earthquakes with intensity of about 9 on the MSK scale with their actual accelerations. The records were taken from the database of the accelerograms described in [11]. Peak accelerations of the considered impacts vary from $2 \mathrm{~m} / \mathrm{s}^{2}$ (Bucharest, Mexico) to $10 \mathrm{~m} / \mathrm{s}^{2}$ (Tabas, Iran), and the prevailing periods range from $0.1 \mathrm{~s}$ to $1.5 \mathrm{~s}$. In the dynamic calculations made by the authors, the considered pier was analyzed with different span structures. The dimensions of such piers are determined by those of the span structures. Therefore, the load can be transferred to the pier from metal single-span flying structures with a weight of up to 100 tons and from continuous reinforced concrete spans weighing more than 12,000 tons. In this case, the oscillation period of the pier with a span structure varies from $0.07 \mathrm{~s}$ to $0.4 \mathrm{~s}$. The ultimate elastic force is considered in fractions of the weight of the span structure and ranges from 0.1 to $0.4 \mathrm{~s}$. The pier loading diagram is shown in Fig.6. There are three sections in it.

In section $0-1$, the pier behavior is elastic until displacements $u<U_{e l}$. In section 1-2, the reinforcement gets plastically deformed, and displacements increase up to $u=U_{\mu}$, after which the pier turns into a mechanism. In section 2-3, the pier body rotates around its side and at point 3 with $u=U_{\text {lim }}$ the pier falls down. The earthquake causes the maximum displacement of the pier $U_{e q}$ marked in the diagram section of 2-3 by point 4 . Where in

$$
K_{1}^{(e q)}=\frac{1}{\mu}=\frac{U_{e l}}{U_{e q}} .
$$

to explain the calculation results, we note two circumstances.

First, the value of $U_{e l}$ and its corresponding coefficient $K_{1}^{(e q)}$ depend on the prevailing input period. However, this dependence should not be monotonous, as was obtained in [5], but should have its peak at a frequency resonant for the structure. If a structure is rigid, i.e. when light spans are placed on the pier and the system's own oscillation frequency is $0.05 \ldots 0.08 \mathrm{~s}$, even strong earthquakes can leave the pier intact.

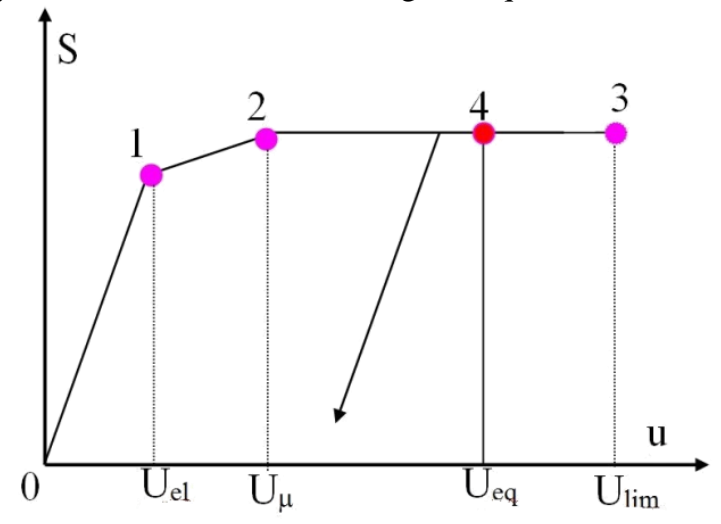

Fig.7. Schematic diagram of pier deformation 
Second, among resonance excitations, the most dangerous input should be considered the one with the higher value of the energy characteristics namely the Arias intensity $\mathrm{I}_{\mathrm{A}}$, the absolute cumulative velocity CAV and the seismic energy density SED.

To estimate the value of $K_{1}^{(e q)}$, the authors considered the following equation

$$
m \ddot{q}+\gamma k m \dot{q}+Q(q, \dot{q})=-m \ddot{y_{0}},
$$

where $m$ is the mass of the span structure, $q$ is the displacement of the top of the pier relative to the base, $k$ is the eigen frequency of the elastic oscillations of the pier with the span structure, $\gamma=0.1$ is the inelastic resistance coefficient of the pier material, which is equal to the double value of critical damping, $Q(q, \dot{q})$ is the pier response in corresponding to the dependence shown in Fig.5, $\ddot{y}_{0}$ is the design accelerogram of the base. Integration of equation (21) was carried out taking into account the piecewise linearity of the system and using an explicit presentation of the solution at each integration step.

\section{Results and discussion}

The calculation results are shown in Fig. 8 for a 12,000-ton fly-over, with the eigen period equal to $0.46 \mathrm{~s}$ for the undamaged pier with a span. Fig. 8 shows the dependence of the plasticity factor $\mu$ on the predominant earthquake period of the accelerogram. Each accelerogram in the picture corresponds to a single point. All in all 88 calculations were carried out using the accelerogram database [5] available to Russian and Chinese coauthors of the paper. In addition to the points in the diagram, an approximating relationship $K_{1}^{(e q)}\left(T_{e q}\right)$ is shown.

As can be seen from the figure, there is no explicit dependence of the coefficient $\mu$ on the prevailing input period found in [5]. This is due to the fact that in [5] the accelerograms are normalized to PGA $=4 \mathrm{~m} / \mathrm{sec}^{2}$. In this case an increase of the plasticity coefficient (a decrease of the reduction coefficient) was observed in [5] for long-period actions. In our studies the PGA is assumed to be actual and it decreases with an increase in the prevailing period, leading to a decrease of the plasticity coefficient. As a result, the average value of the plasticity coefficient is $\mu \approx 4$, and $R_{\mu}=1 / \mu=0.25$.

In general, the value of $\mu$ for a concrete impact is significantly affected by the proximity of the prevailing input period to the eigen structure period. In the case under consideration some strong actions did not cause plastic deformation of the pier at all, although for structures with other periods these actions are very dangerous. There were 12 such actions among 91 used, which turned out dangerous for different structures but not dangerous for pier under consideration.

For illustrating this feature we used the spectrum of plastic deformation work. It is the dependence of the work of plastic deformation force of the elastoplastic system on its initial period. Fig. 9 shows the spectrum of the work of the plastic deformation forces for one of such action (Nortridge earthquake), while the dotted line shows the eigen period of the pier oscillations. The pier eigen frequency is not found in the spectrum frequency band, i.e. the action is not resonant, while resonant actions cause large plastic deformation of the pier and the corresponding value of the coefficient $\mu$. The spectrum of the work of plastic deformation forces for the resonant action (Kobe earthquake) is shown in Fig. 10 


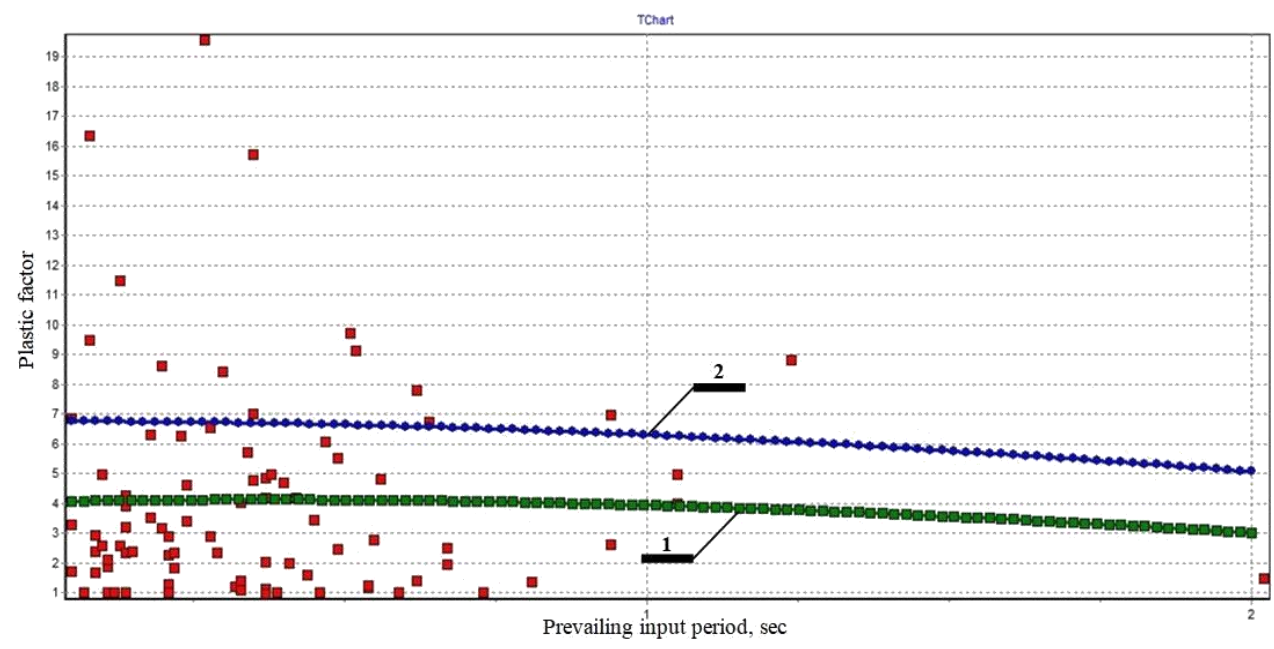

Fig.8. Dependence of the plastic factor on the prevailing input period 1 - average value; $2-\mathrm{Apl}+\sigma$ Apl

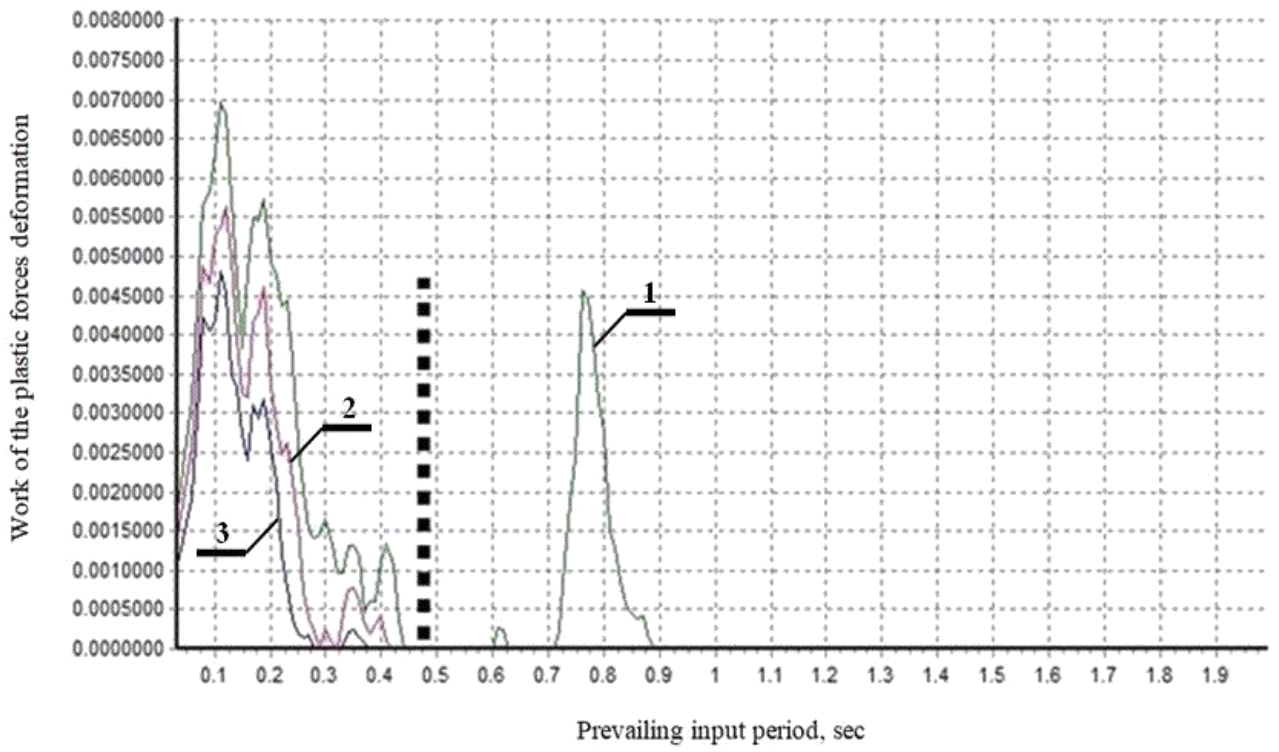

Fig.9. The spectrum of the work of the plastic deformation forces for the Nortridge earthquake $1-\gamma=0.1 ; 2-\gamma=0.15 ; 3-\gamma=0.2$ 


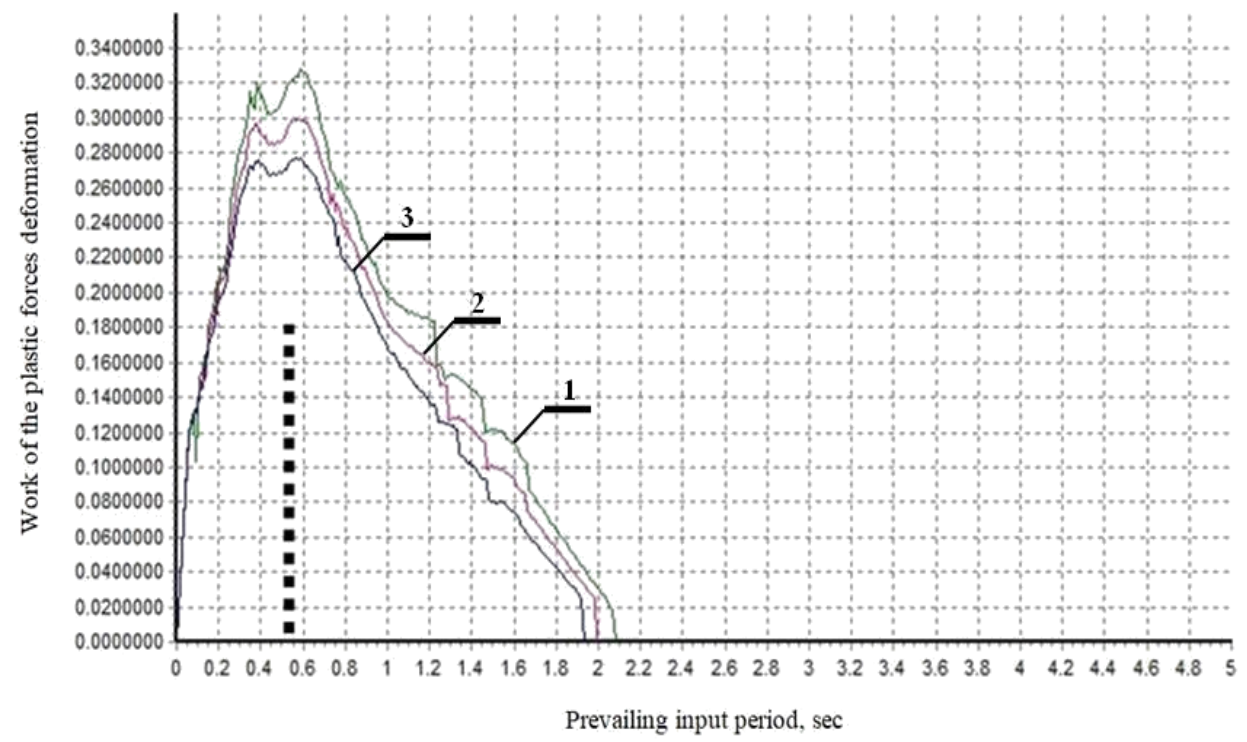

Fig.10. The spectrum of the work of plastic deformation forces for the Kobe earthquake $1-\gamma=0.1 ; 2-\gamma=0.15 ; 3-\gamma=0.2$

Among the actions considered, only 4 turned out to be resonant. However, they caused a weak maximum on the curve $\mu\left(T_{e q}\right)$ in the zone of the natural vibration frequency of the pier, which is shown on the fragment of the $\mu\left(T_{e q}\right)$ dependence (Fig.11).

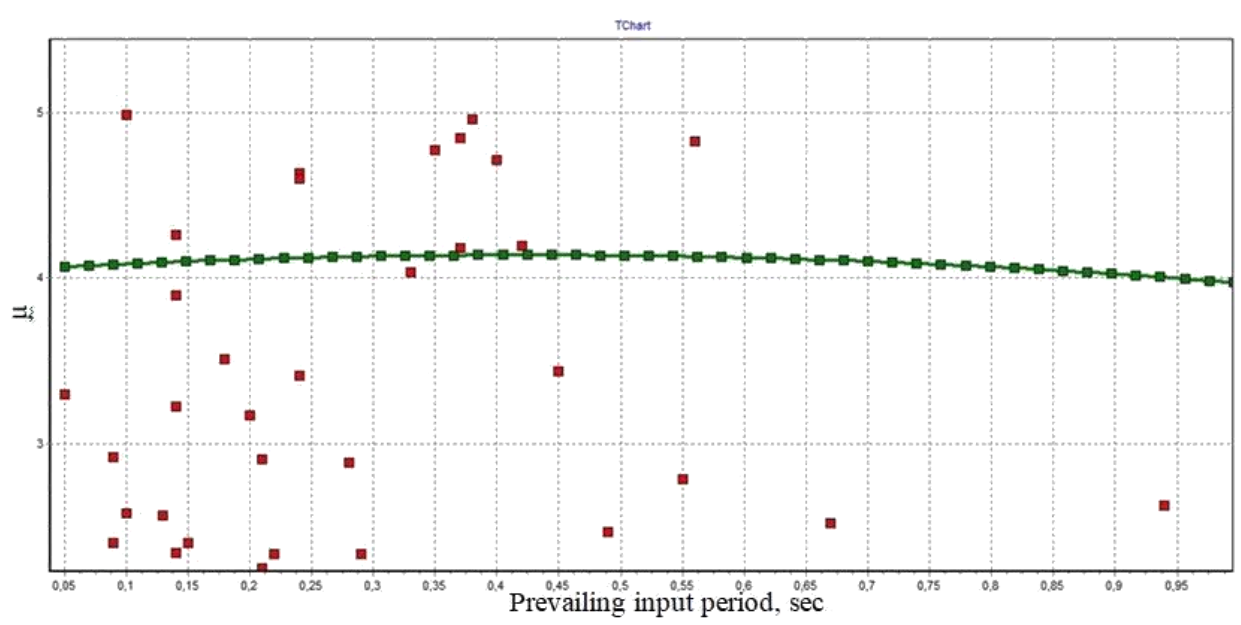

Fig.11. Fragment of dependency $\mu$ (Teq)

Using a packet of calculated accelerograms without a special selection of resonant effects gives a stable average value of the plasticity factor. However, the presence of resonance effects in the packet leads to a large variance in the value of $\mu$. For the case considered, Fig. 7 shows two dependences $-\mu\left(T_{e q}\right)$ and the same value plus its mean-square deviation, i.e. $\mu+\sigma \mu$. It follows that with limited seismological information, as well as with arbitrary inform, action for typical designing, it is necessary to select a packet of 
design accelerograms taking into account the dynamic characteristics of the structure, i.e. to choose resonance actions.

plasticity factor depends essentially on the action spectral composition. In our case it varies from 1 to 20 . This does not allow one to recommend one and the same coefficient for the transition from plastic displacements to the calculated elastic ones. Among many actions only a small amount can be considered dangerous for the structure. In other words, it is impossible to pass from the result of elastic calculation to design displacements and forces using the single plasticity factor $\mu$.

The same result was obtained for the ratio $\frac{S_{\text {cond }}}{S_{\text {calc }}^{(e l)}}$, which is often used for connection elastic and inelastic calculations. This dependence on the predominant accelerogram period is shown in figure 12 with the maximum of this dependence being clearly seen.

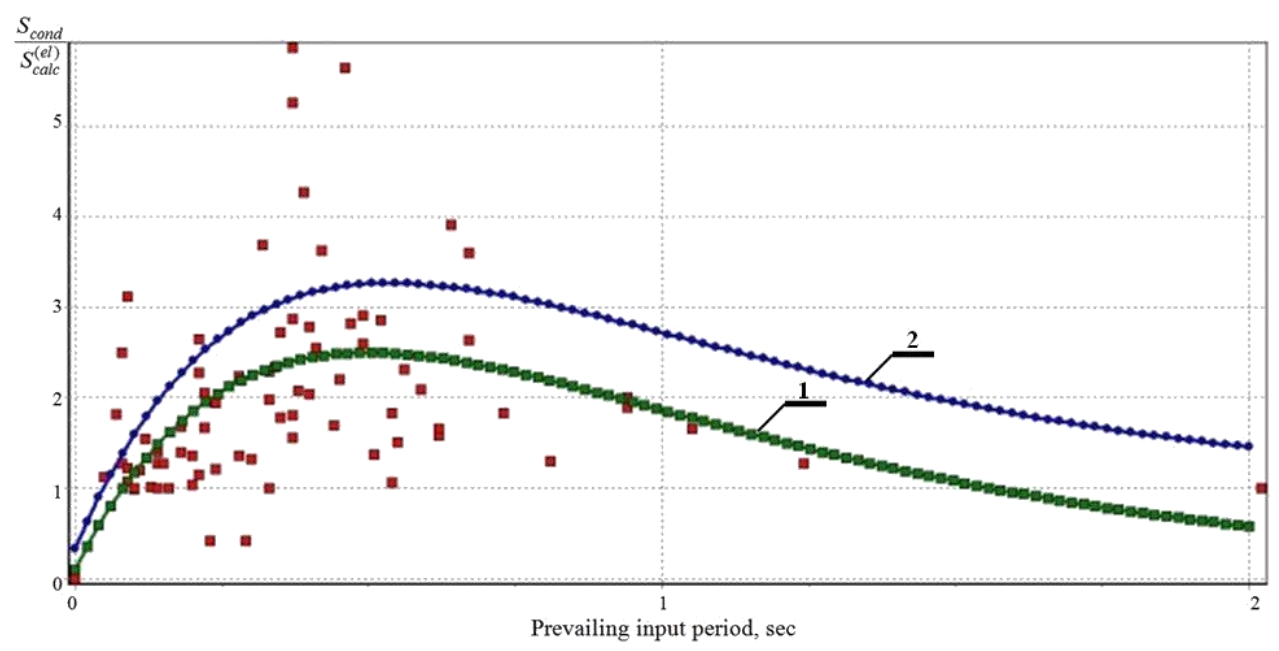

Fig.12. Dependency $\frac{S_{\text {cond }}}{S_{\text {calc }}^{(e l)}}\left(\mathrm{T}_{\mathrm{eq}}\right) 1$ - average value; $2-\frac{S_{\text {cond }}}{S_{\text {calc }}^{(e l)}}+\sigma \frac{S_{\text {cond }}}{S_{\text {calc }}^{(e l)}}$

If we take a certain accelerogram and consider piers with different span structures, we get similar results: the input causes the greatest plastic deformations in structures with the danger oscillation period. This period is somewhat longer than the eigen period of the pier without plastic deformation. In other words, the elastic response spectrum has its peak at higher frequencies than the spectrum of the work of plastic deformation forces.

\section{Conclusions}

The research carried out by the authors allows them to state the following

1. Different approaches to estimating the reduction factor can be proposed. As a rule, its estimation is based on reducing the seismic load in the elastic system to a certain design level in accordance with the formula (3). The authors consider the factor $\mu$ more physical to regard it as the coefficient of reduction of the limiting inelastic displacements to elastic one in accordance with the formula (18). In this case, the main 
normative formula, although reduced to a force form, is kinematic, i.e. it restricts the system displacement.

2. To establish the connection between time-history elastoplastic calculation using earthquake accelerograms and calculation using the RSM, it is necessary to make preliminary calculations of a simplified system model using the design accelerogram. When carrying out such calculations, it is necessary to use an accelerogram that is dangerous for the structure. It is impossible to make the transition from seismic loads obtained on the basis of time-history analysis by accelerograms to design loads using one and the same plasticity factor. This coefficient depends significantly on the spectral composition of the action.

3. From the point of view of structure seismic resistance, it is not the concrete plasticity factor at this or that input that is of interest, but the limiting coefficient of plasticity, the excess of which value leads to the structure destruction

4. Table 1 gives a summary of the coefficients $K_{l}$ for the considered pier. To calculate bearings of this type using the GL, the authors recommend to use the value $K_{l}=$ $K_{1}^{(\lim )}=0.25 \div 0.27$

. This means that the admissible limiting displacements of the pier top are about 4 times greater than its limiting elastic displacements.

The authors are grateful to S. R. Grebenshchikova for her help in preparing the English version of the paper. The work was carried out with the support of the grant of the Russian Foundation for Basic Research No 19-35-90111.

\section{References}

1. I.I. Goldenblat, N.A. Nikolaenko, S.V. Polyakov, S.V. Ulyanov, Models of seismic resistance of structures (Stroiizdat, Moscow, 1979)

2. A.V. Perelmuter, O.V. Kabantsev, Magazine of Civil Engineering 1, 6-14 (2015). DOI:10.5862/MCE.53.1

3. R. Riddell, P. Hidalgo, E. Cruz, Earthquake Spectra 5 (3), 571-590 (1989)

4. F.M. Mazzolani, V. Piluso, Theory and design of seismic resistant steel frames (E\&FN Spon Press, London, 1980)

5. E. Simbort, Engineering and construction magazine 1(27), 44-52 (2012). DOI: 10.5862/MCE.27.6

6. A.S. Khatavkar, A.P. Ghadi, P.F. Barbude, International Journal of computer application 0975-8877 (2015) 12-14 (2015)

7. D. Brahmavrathan, C. Arunkumar, Indian Journal of Science and Technology 9(23) 28 (2016). DOI: 10.17485/ijst/2016/v9i23/95981

8. Y.S. Salem, G. Lomiento, J. Khan, International Congress and Exhibition "Sustainable Civil Infrastructures: Innovative Infrastructure Geotechnology", GeoMEast, Facing the Challenges in Structural Engineering, 55-71 (2017). DOI: 10.1007/978-3-31961914-9_5

9. Y.U. Albert, A.A. Dolgaya, T.V. Ivanova, et. al., Magazine of Civil Engineering 8, 98105 (2017). DOI: 10.18720/MCE.76.9

10. K.W. Campbell, Y. Bozorgnia, Earthquake Spectra 28(2), 457-485 (2012). DOI: $10.1193 / 1.4000012$ 
11. T.V. Ivanova, J. Guan, O.P. Nesterova, S.V. Prokopovich, L.N. Smirnova, A.M. Uzdin, D.A. Ivashintzov, Magazine of Civil Engineering 7, 129-138 (2017). DOI: 10.18720/MCE.75.13

12. N. Newmark, E. Rosenbluet, Foundations of earthquake-resistant construction (Stroiizdat, Moscow, 1980)

13. K. Dunsire, T. O'Neill, M. Denford, J. Leaney, ECBS '05. 12th IEEE International Conference and Workshops 1, 62-69 (2005). DOI: 10.1109/ECBS.2005.66

14. N.K. Manjula, Praveen Nagarajan, T.M. Madhavan Pillai, International Refereed Journal of Engineering and Science (IRJES) 2 (5), 14-19 (2003)

15. Kevin K. F. Wong, Journal of Earthquake Engineering 8(2), 315-334 (2004). DOI: 10.1080/13632460409350491

16. P. Favreau, R.J. Archuleta, Geophysical Research Letters 30(5), 1198-1202 (2013). DOI: 10.1029/2002GL015968 